\title{
Then we characterize primes and composite numbers via divisibility
}

\author{
Ikorong Gilbert ${ }^{1 *}$, Rizzo Karl-Joseph ${ }^{2}$ \\ ${ }^{1}$ Centre de calcul et de recherche; Université Pierre et Marie Curie \\ ${ }^{2}$ LMT-Cachan \\ ${ }^{*}$ Corresponding author E-mail: ikorong@ccr.jussieu.fr
}

Copyright (c)2014 Authors. This is an open access article distributed under the Creative Commons Attribution License, which permits unrestricted use, distribution, and reproduction in any medium, provided the original work is properly cited.

\begin{abstract}
In this paper, we show a Theorem which helps us to characterize prime numbers and composite numbers via divisibility; and we use the characterizations of primes and composite numbers to characterize twin primes, Mersenne primes, even perfect numbers, Sophie Germain primes, Fermat primes, Fermat composite numbers and Mersenne composite numbers (we recall that a logic (non recursive) proof of problems posed by twin primes, Mersenne primes, perfect numbers, Sophie Germain primes, Fermat primes, Fermat composite numbers and Mersenne composite numbers, is given in [12]. [[ Prime numbers are well kwown ( see [15] or [19]) and we recall that a composite number is a non prime number. We recall (see [1] or [2] or [3] or [6] or [9] or [10] or [12] or [13] or [14] or [17]) that a Fermat prime is a prime of the form $F_{n}=2^{2^{n}}+1$, where $n$ is an integer $\geq 0$; and a Fermat composite is a non prime number of the form $F_{n}=2^{2^{n}}+1$, where $n$ is an integer $\geq 1$; it is known that for every $j \in\{0,1,2,3,4\}$, $F_{j}$ is a Fermat prime, and it is also known that $F_{5}$ and $F_{6}$ are Fermat composite. We recall (see [11]) that a prime $h$ is called a Sophie Germain prime, if both $h$ and $2 h+1$ are prime; the first few Sophie Germain primes are $2,3,5,11,23,29,41, \ldots$; it is easy to check that 233 is a Sophie Germain prime. A Mersenne prime (see [6] or [10] or [11] or [16] or [19] or [20] or [21]) is a prime of the form $M_{m}=2^{m}-1$, where $m$ is prime; for example $M_{13}$ and $M_{19}$ are Mersenne prime. A Mersenne composite ( see [7] or [9]) is a non prime number of the form $M_{m}=2^{m}-1$, where $m$ is prime; it is known that $M_{11}$ and $M_{67}$ are Mersenne composite. We also recall (see [4] or [5] or [7] or [8] or [10] or [11] or [18] or [19] or [20] or [22]) that an integer $t$ is a twin prime, if $t$ is a prime $\geq 3$ and if $t-2$ or $t+2$ is also a prime $\geq 3$; for example, it is easy to check that $(881,883)$ is a couple of twin primes. Finally, we recall that Pythagoras saw perfection in any integer that equaled the sum of all the other integers that divided evenly into it (see [7] or [9]). The first perfect number is 6 . It's evenly divisible by 1,2 , and 3 , and it's also the sum of 1,2 , and 3 , [note 28, 496 and 33550336 are also perfect numbers (see [7] or [9])]; and perfect numbers are known for some integers $>33550336$ ]].
\end{abstract}

Keywords: Fermat primes, Fermat composite, Mersenne primes, Mersenne composite, perfect numbers, Sophie Germain primes and twin primes.

\section{Introduction}

This paper is divided into four sections. In section.1, we state and prove the Theorem which implies the characterizations of primes and composite numbers. In section.2, using the Theorem of section.1, we characterize prime 
numbers, composite numbers and we also give two characterizations of twin primes. In section.3, we use the characterizations of section.2 to explicite the Fermat primes, the Fermat composite numbers, the Mersenne primes, the even perfect numbers, the Mersenne composite numbers, the twin primes and the Sophie Germain primes. In section.4, using the Theorem of section.1, we state the general conjecture from which all the results mentioned above follow.

\section{Statement and the proof of the theorem which implies the charac- terizations of primes and composite numbers}

Theorem 1.1. Let $n$ be an integer $\geq 4$. Then, $n+1$ is prime or $n+1$ divides $n$ !.

Before simply proving Theorem 1.1, let us remark the following.

Remark 1.2. Let $n$ be an integer $\geq 4$. If $n+1=p^{2}$ (where $p$ is prime), then $n+1$ divides $n$ !.

Proof. Very easy [it suffices to reason by reduction to absurd].

Proof of Theorem 1.1. If $n+1$ is prime, then the proof is ended. If $n+1$ is not prime, then $n+1$ divides $n$ !. Otherwise (we reason by reduction absurd)

$$
n+1 \text { is not prime and } n+1 \text { does not divide } n \text { ! }
$$

and we observe the following.

Observation 1.1.1. Let $p$ be a prime such that $n+1$ is divisible by $p$ (such a p clearly exists). Then $\frac{n+1}{p}$ is an integer and $\frac{n+1}{p} \leq n$ and $p \leq n$ and $\frac{n+1}{p}=p$.

Indeed, it is immediate that $\frac{n+1}{p}$ is an integer [since $p$ divides $n+1$ ], and it is also immediate that $\frac{n+1}{p} \leq n$ [otherwise, $n+1>n p$; now, remarking that $p \geq 2$ (since $p$ is prime), then the previous two inequalities imply that $n+1>2 n$; so $1>n$ and we have a contradiction, since $n \geq 4$, by the hypotheses]. Clearly $p \leq n$ [otherwise, $p>n$; now, recalling that $n+1$ is divisible by $p$, then the previous inequality implies that $n+1=p$. Recalling that $p$ is prime, then the previous equality clearly says that $n+1$ is prime and this contradicts (1.1.0)]. That being so, to prove Observation 1.1.1, it suffices to prove that $\frac{n+1}{p}=p$. Fact: $\frac{n+1}{p}=p$ [otherwise, clearly $\frac{n+1}{p} \neq p$; now, remarking (by using the previous) that $\frac{n+1}{p}$ is an integer and $\frac{n+1}{p} \leq n$ and $p \leq n$; then it becomes trivial to deduce that $\frac{n+1}{p}$ and $p$ are two different integers such that $\left\{p, \frac{n+1}{p}\right\} \subseteq\{1,2,3, \ldots ., n-1, n\}$. The previous inclusion immediately implies that $p \times \frac{n+1}{p}$ divides $1 \times 2 \times 3 \times \ldots \times n-1 \times n$; therefore $n+1$ divides $n$ !, and this contradicts (1.1.0). So $\left.\frac{n+1}{p}=p\right]$. Observation 1.1.1 follows.

The previous trivial observation made, look at $n+1$; observing (by using Observation 1.1.1) that $p$ is prime such that $\frac{n+1}{p}=p$, clearly

$$
n+1=p^{2} \text {, where } p \text { is prime }
$$

Now using (1.1.2) and Remark 1.2, then it becomes trivial to deduce that $n+1$ divides $n$ !, and this contradicts (1.1.0). Theorem 1.1 follows.

Theorem 1.1 immediately implies the characterizations of prime numbers and composite numbers and twin primes.

\section{The characterizations of prime numbers and composite numbers and twin primes.}

Theorem 2.1. (Characterization of primes). Let $n$ be an integer $\geq 4$ and look at $n+1$. Then the following are equivalent.

(1). $n+1$ is prime.

(2). $n+1$ does not divide $n$ !.

To prove simply Theorem 2.1, we need a Theorem of Euclide.

Theorem 2.2 (Euclide). Let $a, b$ and $c$, be integers such that $a \geq 1, b \geq 1$ and $c \geq 1$. If $a$ divides $b c$ and 
if the greatest common divisor of $a$ and $b$ is 1 , then a divides $c$.

Corollary 2.3. Let $n$ be an integer $\geq 1$; fix once and for all $n$, and look at $n$ !. Now let $p$ be a prime $\geq n+1$; then the greatest common divisor of $n$ ! and $p$ is 1 (in particular, $p$ does not divide $n !)$.

Proof. Immediate, and follows immediately by using Theorem 2.2 and the definition of $n$ !, and by observing that $p$ is a prime $\geq n+1$.

Now, we simply prove Theorem 2.1.

Proof of Theorem 2.1. (1) $\Rightarrow(2)$ ]. Immediate, by remarking that $n+1$ is prime and by using Corollary 2.3 .

$(2) \Rightarrow(1)$. Immediate, by remarking that $n+1$ does not divide $n$ ! and by using Theorem 1.1.

Using Theorem 2.1, then the following corollary and Theorem become immediate.

Corollary 2.4. Let $n$ be an integer $\geq 4$ and look at $n+3$. Then the following are equivalent.

(i). $n+3$ is prime.

(ii). $n+3$ does not divide $(n+2)$ !.

Proof. Immediate, and follows from Theorem 2.1, where we replace $n$ by $n+2$.

Theorem 2.5.0. (Characterization of composite numbers). Let $n$ be an integer $\geq 4$ and look at $n+1$. Then the following are equivalent.

(1). $n+1$ is a composite number.

(2). $n+1$ divides $n$ !.

Proof. Immediate and is a trivial consequence of Theorem 2.1.

Now, using Theorem 2.1 and Corollary 2.4, then we have the following weak characterization of twin primes.

Theorem 2.5.1. (A weak characterization of twin primes). Let $n$ be an integer $\geq 4$ and look at the couple $(n+1, n+3)$. Then the following are equivalent

(a). $\quad(n+1, n+3)$ is a couple of twin primes.

(b). $n+1$ does not divide $n$ ! and $n+3$ does not divide $(n+2)$ !.

Proof. Immediate and is a consequence of Theorem 2.1 and Corollary 2.4.

Theorem 2.6. (A non-weak characterization of twin primes). Let $n$ be an integer $\geq 4$ and look at the couple $(n+1, n+3)$. Then the following are equivalent

(c). $\quad(n+1, n+3)$ is a couple of twin primes.

(d). $n+1$ does not divide $n$ ! and $n+3$ does not divide $n$ !.

To prove simply Theorem 2.6, we need the following two lemmas.

Lemma 2.7. Let $n$ be an integer $\geq 4$. If $n+1$ is prime and if $n+3$ does not divide $n$ !, then $n+3$ is prime.

Proof. Otherwise [we reason by reduction to absurd], clearly

$$
n+1 \text { is prime and } n+3 \text { does not divide } n ! \text { and } n+3 \text { is not prime }
$$

and we observe the following.

Observation 2.7.1. Let $p$ be a prime such that $n+3$ is divisible by $p$ ( such a $p$ clearly exists). Then $p \neq n+3$ and $p \neq n+2$ and $p \neq n+1$ and $p \leq n$. Indeed, it is immediate that $p \neq n+3$ [since $n+3$ is not prime (use (2.7.0)), and since $p$ is prime]. It is also immediate that $p \neq n+2$ and $p \neq n+1[[$ otherwise

$$
p=n+2 \text { or } p=n+1
$$

Now recalling that $p$ divides $n+3$ and using (2.7.1.1), then it becomes trivial to deduce that $n+2$ divides $n+3$ or $n+1$ divides $n+3$. We have a contradiction [since $n \geq 4$ (use the hypotheses), therefore $n+2$ does not divide $n+3$ and $n+1$ does not divide $n+3]$. So $p \neq n+2$ and $p \neq n+1]$. That being so, to prove Observation 2.7.1, it suffices to prove that $p \leq n$. Fact: $p \leq n$ [indeed, recalling that $p$ divides $n+3$, and since we have proved that $p \neq n+3$ and $p \neq n+2$ and $p \neq n+1$, then it becomes trivial to deduce that $p \leq n]$. Observation 2.7 .1 follows.

Observation 2.7.2. Let $p$ be a prime such that $n+3$ is divisible by $p$ ( such a $p$ clearly exists). Then $\frac{n+3}{p}$ is an integer and $\frac{n+3}{p} \leq n$ and $\frac{n+3}{p}=p$.

Indeed, it is immediate that $\frac{n+3}{p}$ is an integer [since $p$ divides $n+3$ ], and it is also immediate that $\frac{n+3}{p} \leq n$ [otherwise, $n+3>n p$; now, remarking that $p \geq 2$ (since $p$ is prime), then the previous two inequalities imply that 
$n+3>2 n$; so $3>n$ and we have a contradiction, since $n \geq 4$, by the hypotheses]. That being so, to prove Observation 2.7.2, it suffices to prove that $\frac{n+3}{p}=p$. Fact: $\frac{n+3}{p}=p$ [otherwise, clearly $\frac{n+3}{p} \neq p$; now, remarking (by using the previous) that $\frac{n+3}{p}$ is an integer and $\frac{n+3}{p} \leq n$, and observing (by using Observation 2.7.1) that $p \leq n$; then it becomes trivial to deduce that $\frac{n+3}{p}$ and $p$ are two different integers such that $\left\{p, \frac{n+3}{p}\right\} \subseteq\{1,2,3, \ldots . ., n-1, n\}$. The previous inclusion immediately implies that $p \times \frac{n+3}{p}$ divides $1 \times 2 \times 3 \times \ldots \times n-1 \times n$; therefore $n+3$ divides $n$ !, and this contradicts (2.7.0). So $\left.\frac{n+3}{p}=p\right]$. Observation 2.7.2 follows.

Observation 2.7.3. Let $p$ be a prime such that $n+3$ is divisible by $p$ ( such a $p$ clearly exists). Then $p \neq 2$. Otherwise, $p=2$; now, observing ( by using Observation 2.7.2) that $\frac{n+3}{p}=p$, then the previous two equalities immediately imply that $\frac{n+3}{2}=2$; clearly $n+3=4$ and so $n=1$. We have a contradiction, since $n \geq 4$, by the hypotheses. Observation 2.7.3 follows.

Observation 2.7.4. Let $p$ be a prime such that $n+3$ is divisible by $p$ ( such a $p$ clearly exists). Then $p \neq 3$. Otherwise, $p=3$; now, observing ( by using Observation 2.7.2) that $\frac{n+3}{p}=p$, then the previous two equalities immediately imply that $\frac{n+3}{3}=3$; clearly $n+3=9$ and so $n=6$. Clearly $n+3$ divides $n$ !, and this contradicts (2.7.0) (note that $n+3$ divides $n$ !, since $n+3=9, n=6$ and 9 divides 6!). Observation 2.7.4 follows.

Observation 2.7.5. Let $p$ be a prime such that $n+3$ is divisible by $p$ ( such a $p$ clearly exists). Then $p \geq 5$ and $\frac{2(n+3)}{p}$ is an integer and $\frac{2(n+3)}{p} \leq n$ and $\frac{2(n+3)}{p} \neq p$.

Clearly $p \geq 5$ [indeed, observe that $p \neq 2$ (by Observation 2.7.3) and $p \neq 3$ (by Observation 2.7.4); now using the previous and the fact that $p$ is prime, then it becomes trivial to deduce that $p \geq 5$ ]. Indeed, it is immediate that $\frac{2(n+3)}{p}$ is an integer [since $p$ divides $n+3$ ], and it is also immediate that $\frac{2(n+3)}{p} \leq n$ [otherwise, $2(n+3)>n p$; now, remarking ( by using the previous) that $p \geq 5$, then the previous two inequalities imply that $2(n+3)>5 n$; so $6>3 n$ and therefore $2>n$. We have a contradiction, since $n \geq 4$, by the hypotheses]. That being so, to prove Observation 2.7.5, it suffices to prove that $\frac{2(n+3)}{p} \neq p$. Fact: $\frac{2(n+3)}{p} \neq p$ [otherwise, clearly $\frac{2(n+3)}{p}=p$; now, remarking (by using Observation 2.7.2) that $\frac{n+3}{p}=p$, then the previous two equalities immedialy imply that $\frac{2(n+3)}{p}=\frac{n+3}{p}$; so $2(n+3)=n+3$ and this last equality is clearly impossible. So $\left.\frac{2(n+3)}{p} \neq p\right]$. Observation 2.7.5 follows.

The previous trivial observations made, look at $n+3$; observing (by using Observation 2.7.5) that $\frac{2(n+3)}{p}$ is an integer and $\frac{2(n+3)}{p} \leq n$ and $\frac{2(n+3)}{p} \neq p$, and remarking (by using Observation 2.7.1) that $p \leq n$, then it becomes trivial to deduce that $\frac{2(n+3)}{p}$ and $p$ are two different integers such that $\left\{p, \frac{2(n+3)}{p}\right\} \subseteq\{1,2,3, \ldots . ., n-1, n\}$. The previous inclusion immediately implies that $p \times \frac{2(n+3)}{p}$ divides $1 \times 2 \times 3 \times \ldots \times n-1 \times n$; therefore $2(n+3)$ divides $n$ !; clearly $n+3$ divides $n$ ! and this contradicts (2.7.0). Lemma 2.7 follows.

Lemma 2.8. Let $n$ be an integer $\geq 4$. If $n+1$ is prime and if $n+3$ does not divide $n$ !, then $(n+1, n+3)$ is a couple of twin primes.

Proof.Indeed, since $n+1$ is prime and $n+3$ does not divide $n$ !, then Lemma 2.7 implies that $n+3$ is prime; therefore $(n+1, n+3)$ is a couple of twin primes.

The previous simple two lemmas made, we now prove Theorem 2.6.

Proof of Theorem 2.6. $(c) \Rightarrow(d)$ ]. Immediate. Indeed, since $(n+1, n+3)$ is a couple of twin primes, clearly $n+1$ does not divide $n$ ! (by observing that $n+1$ is prime and by using Theorem 2.1) and clearly $n+3$ does not divide $n$ ! [ otherwise $n+3$ divides $n$ ! and we trivially deduce that

$$
n+3 \text { divides }(n+2) \text { ! }
$$

Now using (2.6.1) and Corollary 2.4, then it becomes immediate to deduce that $n+3$ is not prime. A contradiction, since $(n+1, n+3)$ is a couple of twin primes, and in particular $n+3$ is prime].

$(d) \Rightarrow(c)$ ]. Immediate. Indeed, if $n+1$ does not divide $n$ ! and if $n+3$ does not divide $n$ !, clearly $n+1$ is prime (by observing that $n+1$ does not divide $n$ ! and by using Theorem 2.1) and clearly $(n+1, n+3)$ is a couple of twin primes [ by remarking that $n+1$ is prime (use the previous) and by observing that $n+3$ does not divide $n$ ! and by using Lemma 2,8].

From Theorem 2.1 and Theorem 2.5.0 and Theorem 2.5.1 and Theorem 2.6, we deduce explicite characterizations of Fermat primes, Fermat composite, Mersenne primes, Mersenne composite, Sophie Germain primes and even perfect numbers; and moreover, we also give explicite sets containing the Fermat primes, the Fermat composite numbers, the Mersenne primes, the Mersenne composite numbers, the twin primes and the Sophie Germain primes. 


\section{Applications of Theorem 2.1 and Theorem 2.5.0 and Theorem 2.5.1 and Theorem 2.6, to the Fermat composite numbers, the Fermat primes, the Mersenne primes, the Mersenne composite numbers , the twin primes, the Sophie Germain primes and the even perfect numbers}

Theorem 3.1. ( A characterization of Fermat primes). Let $n$ be an integer of the form $n=2^{2^{n^{\prime}}}$, where $n^{\prime}$ is integer $\geq 1$. Then we have the following two properties.

(3.1.1). $n+1$ is a Fermat prime, if and only if, $n+1$ does not divide $n$ !.

(3.1.2). For every integer $x \geq n+1$, the set $\mathcal{F}(x)=\{n+1 ; x \geq n+1$ and $n+1$ does not divide $n$ ! $\}$ is exactly the set of all Fermat primes $\leq x$ and $\geq 5$.

Proof. Property (3.1.1) is immediate and is a trivial consequence of Theorem 2.1. Property (3.1.2) is also immediate and is a trivial consequence of property (3.1.1).

Theorem 3.2. ( A characterization of Fermat composite numbers). Let $n$ be an integer of the form $n=2^{2^{n^{\prime}}}$, where $n^{\prime}$ is integer $\geq 1$. Then we have the following two properties.

(3.2.1). $n+1$ is a Fermat composite number, if and only if, $n+1$ divides $n$ !.

(3.2.2). For every integer $x \geq n+1$, the set $\mathcal{F C O}(x)=\{n+1 ; x \geq n+1$ and $n+1$ divides $n$ ! $\}$ is exactly the set of all Fermat composite numbers $\leq x$.

Proof. Property (3.2.1) is immediate and is a trivial consequence of Theorem 2.5.0. Property (3.2.2) is also immediate and is a trivial consequence of property (3.2.1).

Theorem 3.3. ( A characterization of Mersenne primes). Let $n$ be an integer of the form $n=2^{n^{\prime}}$, where $n^{\prime}$ is prime $\geq 3$. Then we have the following two properties.

(3.3.1). $n-1$ is a Mersenne prime, if and only if, $n-1$ does not divide $(n-2)$ !.

(3.3.2). For every fixed integer $x \geq n-1, \mathcal{M}(x)=\{n-1 ; x \geq n-1$ and $n-1$ does not divide $(n-2) !\}$ is exactly the set of all Mersenne primes $\leq x$ and $\geq 7$.

Proof. Property (3.3.1) is immediate and is a trivial consequence of Theorem 2.1. Property (3.3.2) is also immediate and is a trivial consequence of property (3.3.1).

Theorem 3.4. ( A characterization of Mersenne composite numbers). Let $n$ be an integer of the form $n=2^{n^{\prime}}$, where $n^{\prime}$ is prime $\geq 3$. Then we have the following two properties.

(3.4.1). $n-1$ is a Mersenne composite number, if and only if, $n-1$ divides $(n-2)$ !.

(3.4.2). For every fixed integer $x \geq n-1, \mathcal{M C}(x)=\{n-1 ; x \geq n-1$ and $n-1$ divides $(n-2) !\}$ is exactly the set of all Mersenne composite numbers $\leq x$.

Proof. Property (3.4.1) is immediate and is a trivial consequence of Theorem 2.5.0. Property (3.4.2) is also immediate and is a trivial consequence of property (3.4.1).

Theorem 3.5. ( A characterization of Sophie Germain primes). Let $n$ be an integer of the form $n=p-1$, where $p$ is a prime $\geq 5$. Then we have the following two properties.

(3.5.1). $n+1$ is a Sophie Germain prime, if and only if, $2(n+1)+1$ does not divide $2(n+1)$ !.

(3.5.2). For every fixed integer $x \geq n+1, \mathcal{H}(x)=\{n+1 ; x \geq n+1$ and $2(n+1)+1$ does not divide $2(n+1) !\}$ is exactly the set of all Sophie Germain primes $\leq x$ and $\geq 5$.

Proof. Property (3.5.1) is immediate and is a trivial consequence of Theorem 2.1 and the definition of the Sophie Germain primes. Property (3.5.2) is also immediate and is a trivial consequence of property (3.5.1).

Twin primes were characterized in Section.2. Now the following Theorem presents the set of twin primes for a given integer.

Theorem 3.6. ( $A$ set of twin primes). Let $n$ be an integer $\geq 4$. Then we have the following three properties.

(3.6.1).For every integer $x \geq n+3, \mathcal{T}(x)=\{(n+1, n+3) ; n+1$ does not divide $n$ !

and $n+3$ does not divide $(n+2)$ ! $\}$ is exactly the set of all twin primes $\leq x$ and $\geq 5$.

(3.6.2).For every integer $x \geq n+3, \mathcal{T}^{\prime}(x)=\{(n+1, n+3) ; n+1$ does not divide $n$ !

and $n+3$ does not divide $n !\}$ is exactly the set of all twin primes $\leq x$ and $\geq 5$. 
(3.6.3). For every fixed integer $x \geq n+3, \mathcal{T}(x)=\mathcal{T}^{\prime}(x)$, where $\mathcal{T}(x)$ is defined in the property (3.6.1) and where $\mathcal{T}^{\prime}(x)$ is defined in the property (3.6.2).

Proof. Property (3.6.1) is immediate and is a trivial consequence of Theorem 2.5.1 and the definition of twin primes. Property (3.6.2) is also immediate and is a trivial consequence of Theorem 2.6 and the definition of twin primes. Property (3.6.3) is immediate, and is a trivial consequence of property (3.6.1) and property (3.6.2).

Now using Theorem 3.3, then the following last Theorem becomes immediate.

Theorem 3.7. (Characterization of even perfect numbers). Let $m$ be an integer $\geq 3$ and look at $2^{m}-1$. Then the following are equivalent.

(1). $2^{m-1}\left(2^{m}-1\right)$ is an even perfect number.

(2). $2^{m}-1$ does not divide $\left(2^{m}-2\right)$ !.

To prove Theorem 3.7, we need the following known Theorem

Theorem 3.8. (Euler). The following are equivalent.

(1). $e$ is an even perfect number.

(2). $e$ is of the form $2^{m-1}\left(2^{m}-1\right)$, where $2^{m}-1$ is prime.

Proof of Theorem 3.7. Immediate and follows by using Theorem 3.7 and Theorem 3.3.

\section{Statement of the general conjecture from which all the previous results follow}

Indeed, using Theorem 1.1, then it becomes a little natural to us to conjecture the following:

Conjecture 4.1. For every integer $j \geq 0$ and for every integer $n \geq 4+j$; then, $n+1+j$ is prime or $n+1+j$ divides $n$ !.

It is immediate that the previous conjecture implies Theorem 1.1, and therefore, all the results proved in section.1 and section.2 and section.3, are only an immediate consequence of Conjecture 4.1. Moreover, if Conjecture 4.1 is true, then, puting $j=n-4$ ( $n$ can be very large), it immediately follows that $2 n-3$ is prime or $2 n-3$ divides $n$ !.

\section{References}

[1] Dickson. Theory of Numbers (History of Numbers. Divisibity and primality) Vol 1. Chelsea Publishing Company. New York, N.Y (1952). Preface.III to Preface.XII.

[2] Dickson. Theory of Numbers (History of Numbers. Divisibity and primality) Vol 1. Chelsea Publishing Company. New York , N.Y (1952)

[3] G.H Hardy, E.M Wright. An introduction to the theory of numbers. Fith Edition. Clarendon Press. Oxford.

[4] Ikorong Anouk Gilbert Nemron An alternative reformulation of the Goldbach conjecture and the twin primes conjecture. Mathematicae Notae. Vol XLIII (2005). 101 - 107.

[5] Ikorong Anouk Gilbert Nemron. Around The Twin Primes Conjecture And The Goldbach Conjecture I. Tomul LIII, Analele Stiintifice Ale Universitatii "Sectiunea Matematica". (2007). 23 - 34.

[6] Ikorong Anouk Gilbert Nemron. An original symposium over the Goldbach conjecture, The Fermat primes, The Fermat composite numbers conjecture, and the Mersenne primes conjecture .Mathematicae Notae. Vol XLV (2008). $31-39$.

[7] Ikorong Anouk Gilbert Nemron. An original abstract over the twin primes, the Goldbach Conjecture, the Friendly numbers, the perfect numbers, the Mersenne composite numbers, and the Sophie Germain primes. Journal of Discrete Mathematical Sciences And Cryptography; Taru Plublications; Vol.11; Number.6, (2008). 715 - 726.

[8] Ikorong Anouk Gilbert Nemron. Playing with the twin primes conjecture and the Goldbach conjecture. Alabama Journal of Maths; Spring/Fall 2008. $47-54$.

[9] Ikorong Anouk Gilbert Nemron. A Glance At A Different Kinds Of Numbers. International Journal Of Mathematics And Computer Sciences. Vol.4, No.1, 2009. 43 - 53. 
[10] Ikorong Anouk Gilbert Nemron. Runing With The Twin Primes, The Goldbach Conjecture, The Fermat Primes Numbers, The Fermat Composite Numbers, And The Mersenne Primes; Far East Journal Of Mathematical Sciences; Volume 40, Issue 2, May2010, $253-266$.

[11] Ikorong Anouk Gilbert Nemron. Speech around the twin primes conjecture, the Mersenne primes conjecture, and the Sophie Germain primes conjecture; Journal Of Informatics And Mathematical Sciences; Volume 3, 2011, No 1, 31 - 40.

[12] Ikorong Anouk Gilbert Nemron. A Proof Of Eight Famous Number Theory Problems And Their Connection To The Goldbach Conjecture. South Asian Journal Of Mathematics; Vol1 (3); 2011, 87 - 105.

[13] Ikorong Anouk Gilbert Nemron. Nice Rendez Vous With Primes And Composite Numbers. South Asian Journal Of Mathematics; Vol1 (2); 2012, $68-80$.

[14] Ikorong Anouk Gilbert Nemron. Placed Near The Fermat Primes And The Fermat Composite Numbers. International Journal Of Research In Mathematic And Apply Mathematical Sciences; Vol3; 2012, 72 - 82.

[15] Ikorong Anouk Gilbert Nemron. Around Prime Numbers And Twin Primes. Theoretical Mathematics And Applications; Vol3, No1; 2013, $211-220$.

[16] Ikorong Anouk Gilbert Nemron. Invited By The Mersenne Primes, The Mersenne Composite Numbers, And The Perfect Numbers. Will appear in Theoretical Mathematics And Applications; Vol3, No2; 2013.

[17] Ikorong Anouk Gilbert Nemron. Meeting With Primes And Composite Numbers. Will Appear In Asian Journal of Mathematics and Applications; 2013.

[18] Maria Suzuki. Aternative Formulations of the Twin Primes Problem. The American Mathematical Monthly. Volume 107. Juanuary 2000. $55-56$.

[19] P. Dusart. Inegalits Explicites pour $\psi(X), \theta(X), \pi(X)$, et les Nombres Premiers. C.R.Math.Rep.Acad.Sci. Canada. Vol. 21 (1). 1999. 53 - 59.

[20] Paul Hoffman. Erdös, l'homme qui n'aimait que les nombres. Editions Belin, (2000). 30 - 49.

[21] Paul Hoffman. The man who loved only numbers. The story of Paul Erdös and the search for mathematical truth. 1998.30 - 49 .

[22] O. Ramare et R. Rumely. Primes in Arithmetic Progressions. Math.Comp. (213). 65. 1996. 397 - 425. 